\title{
SPACE WEATHER STUDIES OF IONOLAB GROUP
}

Feza Arikan, Umut Sezen, Cenk

Toker, Harun Artuner, Gurhan Bulu,

Uygar Demir, Esra Erdem

Hacettepe University

Ankara, Turkey

arikan@hacettepe.edu.tr,

U.Sezen@ee.hacettepe.edu.tr,

cenk.toker@ee.hacettepe.edu.tr,

artuner@hacettepe.edu.tr,

demir@ee.hacettepe.edu.tr,

esraerd@gmail.com

\author{
Orhan Arikan, Hakan Tuna \\ Bilkent University \\ Ankara, Turkey \\ oarikan@ee.bilkent.edu.tr, \\ htuna@bilkent.edu.tr \\ Secil Karatay \\ Kastamonu University \\ Kastamonu, Turkey \\ skaratay@,kastamonu.edu.tr
}

\author{
Tamara L. Gulyaeva \\ IZMIRAN \\ Moscow, Russia \\ gulyaeva@izmiran.ru \\ Zbysek Mosna \\ Institute of Atmospheric Physics, \\ Academy of Sciences, \\ Prague, Czech Republic \\ zbn@ufa.cas.cz
}

\begin{abstract}
IONOLAB is an interdisciplinary research group dedicated for handling the challenges of near earth environment on communication, positioning and remote sensing systems. IONOLAB group contributes to the space weather studies by developing state-of-the-art analysis and imaging techniques. On the website of IONOLAB group, www.ionolab.org, four unique space weather services, namely, IONOLAB-TEC, IRI-PLAS2015, IRI-PLAS-MAP and IRI-PLAS-STEC, are provided in a user friendly graphical interface unit. Newly developed algorithm for ionospheric tomography, IONOLAB-CIT, provides not only 3-D electron density but also tracking of ionospheric state with high reliability and fidelity. The algorithm for ray tracing through ionosphere, IONOLAB-RAY, provides a simulation environment in all communication bands. The background ionosphere is generated in voxels where IRI-Plas electron density is used to obtain refractive index. One unique feature is the possible update of ionospheric state by insertion of Total Electron Content (TEC) values into IRI-Plas. Both ordinary and extraordinary paths can be traced with high ray and low ray scenarios for any desired date, time and transmitter location. 2D regional interpolation and mapping algorithm, IONOLABMAP, is another tool of IONOLAB group where automatic TEC maps with Kriging algorithm are generated from GPS network with high spatio-temporal resolution. IONOLAB group continues its studies in all aspects of ionospheric and plasmaspheric signal propagation, imaging and mapping.
\end{abstract}

Keywords-ionosphere; IRI; IONOLAB; total electron content; computerized ionospheric tomography; mapping; ray tracing; GPS

\section{INTRODUCTION}

The Space Weather (SW) is defined by the coupling of solar activity into the Earth's magnetosphere and the variability that is observed in the surrounding atmosphere (from plasmasphere down to ozone layer) with the solar wind - magnetosphere - ionosphere storm and substorm impact on man-made structures and man-made electromagnetic signals [1].

The ionospheric weather is one of the key components of the SW affecting the ground-based and space-based satellites systems for communication, navigation and positioning. The solar magnetic field and coronal mass ejections are proven to be the most important contributors of solar wind, and thus, they represent factors of risk for the ionospheric weather from above. The lithosphere-atmosphere-ionosphere coupling provide evidence of a risk of impact of seismic activity on the ionosphere from below through acoustic and gravitational waves registered as fluctuations of Total Electron Content (TEC) and critical F2 layer peak parameters of ionosphere [1].

Comprehensive studies of the ionospheric day-to-day variability reveal comparable effects in the ionosphere induced by the solar and geomagnetic storms and substorms and the meteorological natural hazards. Investigation of the risk factors affecting man-made devices and electromagnetic signals in the ionosphere from the solar, magnetospheric and seismic sources presents a major challenge for researchers from all disciplines.

IONOLAB group is an interdisciplinary group where researchers from electrical and computer engineering, ionospheric physics and geodesy have come together to develop state-of-the-art tools to investigate, model, image and predict the structure of ionosphere using GPS and ionosonde data [2], [3]. IONOLAB group is in close collaboration with IZMIRAN Institute from Russian Federation and Atmospheric Sciences Institute of Czech Republic. Since 1995, the studies are IONOLAB group have been sponsored by the grants from The Scientific and Technological Research Council of Turkey (TÜBITAK). There are four completed projects and four continuing projects since 2006 with more than 40 journal papers and 150 conference presentations.

In this paper, the main online space weather services and products of IONOLAB group, namely, IONOLAB-TEC, IRIPLAS, IRI-PLAS-MAP, IRI-PLAS-STEC, IONOLAB-CIT, IONOLAB-MAP, IONOLAB-RAY are introduced briefly in the following section. The paper ends with a discussion and future direction of IONOLAB group studies. 


\section{ONLINE SPACE WEATHER SERVICES}

The online SW services of IONOLAB group are provided from www.ionolab.org website. The user needs to register once by giving an email account. Below is the brief explanation of these services.

\section{A. IONOLAB-TEC}

Total Electron Content (TEC) is defined as the line integral of electron density on a ray path. The unit of TEC is TECU and $1 \mathrm{TECU}=10^{16} \mathrm{el} / \mathrm{m}^{2}$. TEC is the projection of electron density in the vertical direction and it is an observable that indicates the spatio-temporal variability of ionosphere. Global Positioning System (GPS) provides a cost-efficient means of estimating TEC with its dual-frequency earth based receivers. The total number of electrons between the receiver and GPS satellite in a cylinder with $1 \mathrm{~m}^{2}$ base are is defined as Slant TEC (STEC) [2], [3], [4].

The relatively easy model of GPS-STEC estimation becomes a hard task due to the 'Differential Code Bias' terms in the STEC equation due to the receiver and satellite [4], [5]. IONOLAB-TEC is the TEC estimation routine developed in IONOLAB group since 2001 [4]-[6]. In its current version, IONOLAB-TEC algorithm can automatically parse any version of GPS receiver files in the Receiver INdependent EXchange (RINEX) format with any time sampling interval and any RINEX package length. The satellite DCB is obtained from IONospheric Exchange (IONEX) files provided by International International Global Navigation Satellite Systems (GNSS) Service (IGS) (ftp://igscb.jpl.nasa.gov) analysis centers. Receiver DCB is computed in a special routine automatically in the algorithm using IONOLAB-BIAS method [7]. The output TEC can be delivered in a graphical interface or can be provided in an excel file. Multiple days or multiple stations can be processed in the same batch. IONOLAB-TEC can also be downloaded in an executable form for batch operations. IONOLAB-TEC can estimate GPSTEC from any station in high, equatorial or mid latitude region, both for disturbed and nondisturbed days of ionosphere with high reliability, accuracy and robustness [2]-[7].

\section{B. IRI-PLAS}

International Reference Ionosphere (IRI) is the most acknowledged climatic model of ionosphere that provides electron density profile and hourly, monthly median values of critical layer parameters of the ionosphere for a desired location, date and time between 60 to $2,000 \mathrm{~km}$ altitude [8]. IRI is accepted as the International Standard Ionosphere model as given in [9]. Recently, the IRI model is extended to the GPS satellite orbital range of $20,000 \mathrm{~km}$ and GPS-TEC can be input to the model to update the state of ionosphere [10]. The new version is called IRI-Plas and it can be obtained from http://ftp.izmiran.ru/pub/izmiran/SPIM/. Due to its extension to GPS satellite orbital height, IRI-Plas is also widely used as a physical background model in Computerized Ionospheric Tomography (CIT) both for regional and global reconstruction of electron density.

A user-friendly online version of IRI-Plas is provided at www.ionolab.org as a space weather service. The user can enter the basic inputs of location, date, time. Multiple set of inputs can be entered in a batch and the program is run by just clicking the button, 'RUN'. The output is provided with a preamble of location in geographic and geomagnetic coordinates, critical parameter values for the ionospheric layers, propagation parameters. The electron density and electron and ion temperature values are also provided with respect to height according to the demand of the user. The optional inputs of foF2, propagation model inputs, geomagnetic indices and storm model options are provided with a graphical user interface.

The most important contribution of IRI-Plas to the ionospheric climatic model is the input of GPS-TEC. Currently, IRI model provides TEC as an output as the total number of electrons from the lowest layer of ionosphere to the $2.000 \mathrm{~km}$ in altitude. Although there is an option for IRI to input TEC, the model does not change any of the main parameters with respect to the TEC input by the user. As mentioned in the previous subsection, in recent years, GPS system became the most important source for TEC estimation. IRI-Plas can input GPS-TEC, foF2 and hmF2 and it scales the ionospheric parameters including foF2 with respect to the input. This way the empirical and deterministic structure of ionospheric electron density is updated to the current solar and geomagnetic conditions. The input of TEC can be done automatically using the Global Ionospheric Maps (GIM) produced by IGS analysis centers. By clicking on the 'GIMTEC' button, a world map with GPS receivers in IGS and EUREF network are shown. The user can choose the GPS station and individual GIM-TEC estimates of IGS analysis centers are provided with a pop-up window. The user can automatically choose to input any TEC value or an average value of TEC estimates by just clicking appropriate button. This is a unique service and reduces the overhead of accessing the GIM and extracting the correct value for the given coordinate.

The user can also input TEC, foF2 and hmF2 values or, if desired GPS-TEC from Global Ionospheric Maps (GIM) can be automatically ingested similar to the application of IRIPlas-MAP at www.ionolab.org. IONOLAB-IRI-Plas will provide selected ionospheric parameters according to the updated state of ionosphere in a user-friendly fashion.

\section{IRI-PLAS-MAP}

The online IRI-PLAS-MAP service of IONOLAB group assimilates the GIM-TEC values into IRI-Plas model for a user defined region, date and time period, thus integrating the ionospheric dynamics into the model [11]. The user can choose to compute IRI-PLAS-TEC, -foF2, -hmF2 or the Windex (an indicator of ionospheric disturbance) by assimilating either one-hour time resolution UHRG GIM or two-hour time resolution JPL GIM. IRI-PLAS-MAP can provide hmF2, foF2, TEC and W-index maps in both IGS standard IONEX text file format and graphical format. The spatial resolution of IRI-PLAS-MAP is the same as GIM. The desired IRI-PLASMAPs can be downloaded through the link that is sent to the user e-mail when the files are ready. This unique service 
allows the users from all disciplines to observe, model and monitor ionospheric parameters of SW.

\section{IRI-PLAS-STEC}

GPS-STEC contains the space weather variability, yet the estimates are prone to measurement and instrument errors that are not related to the physical structure of the ionosphere. Although the IRI-Plas can be used as a background model in CIT, computation of STEC from IRI-Plas is a tedious task for researchers due to extensive geodetic calculations and IRI-Plas runs. An important SW service from IONOLAB group is the online computation of STEC from IRI-Plas as IRI-PLASSTEC at www.ionolab.org [12]. The IRI-PLAS-STEC can be computed online for a desired location, date, hour, elevation and azimuth angle. The user-friendly interface also provides means for computation of IRI-PLAS-STEC for a desired location and date to indicate the variability in hour of the day, elevation or azimuth angles. The desired location can be chosen as a GPS receiver in IGS or EUREF networks. Also, instead of specifying elevation and azimuth angles, the user can directly choose from the GPS satellites and obtain IRIPLAS-STEC for a desired date and/or hour. The computed IRI-PLAS-STEC values are presented directly on the screen or via email as both text and plots. If the computation time is longer than a certain period, the output data files are sent to the user via registered email of the user. IRI-PLAS-STEC proved itself as a reliable means as an indicator or precursor of geomagnetic, gravitational and seismic disturbances in the ionosphere [13].

\section{2-D AND 3-D IMAGING OF IONOSPHERE: IONOLAB-MAP AND IONOLAB-CIT}

IONOLAB-MAP is an automatic 2-D reconstruction algorithm that is developed for spatio-temporal interpolation of TEC with high resolution [14]. The algorithm utilizes isotropic Ordinary Kriging (OK) algorithm for high latitude and equatorial regions and Universal Kriging with linear trend (UK1) for midlatitude regions [15]. The theoretical semivariogram function that represents the spatial correlation structure of TEC is chosen to be from the Matern Family for the first time in TEC mapping. The trend model parameters are obtained from TEC samples in closed form and the Matern parameters are estimated using Particle Swarm Optimization (PSO). The automatic structure enables TEC maps to be obtained with any desired time interval. The algorithm is also capable of automatic assimilation of GIM-TEC to reconstruct missing data. GIM-TEC is also used to frame the GPS network and this way, increase the reliability of reconstruction by reducing the error variability on the borders of GPS network region.

IONOLAB-MAP is a versatile tool where user only enters date, time, list and location of GPS stations in the network and the input data directory address. In the initial stage of the algorithm, TEC values are resampled over a lowresolution grid by Inverse Distance Weighting (IDW) algorithm. This step ensures the uniformity in the data for the experimental semivariogram calculation. The theoretical semivariogram function is obtained by fitting the experimental values to a Matern family. The parameters of Matern function are estimated using PSO algorithm. The closer grid points within $150 \mathrm{~km}$ radius are weighed with a nonuniform function to overcome the disadvantages of IDW. With Kriging algorithms, the TEC is mapped in a denser grid. Both the first rough grid and the second denser grid sizes are chosen automatically by IONOLAB-MAP to ensure highest spatial resolution and reliability of interpolation algorithm. The TEC map output can be provided both in a graphical form or it can be saved automatically in a user-specified directory. IONOLAB-MAP is a unique algorithm that provides high spatio-temporal TEC maps for any region.

3-D imaging of the electron density distribution in the ionosphere is a crucial task for investigating the ionospheric variability on satellite signals. Reconstruction of ionospheric electron density using GPS signals is called Computerized Ionospheric Tomography (CIT) [16], [17]. The main disadvantage of CIT is sparse and highly nonuniform distribution of GPS-STEC. Standard tomographic reconstruction techniques in the literature are not accurate or reliable enough to represent the full complexity of variable ionosphere. The model based electron density distributions are produced according to the general trends of ionosphere, and these distributions do not agree with measurements, especially for geomagnetically active hours. IONOLAB-CIT is a regional 3-D electron density distribution reconstruction method where GPS-STEC is assimilated into IRI-Plas by adding a planar perturbation surface to physical model based F2 layer critical frequency, foF2, and maximum ionization height, hmF2, [17]. IONOLAB-CIT is based on an iterative optimization framework that tracks the deviations from the ionospheric model in terms of foF2 and hmF2 resulting from the comparison of IRI-PLAS-STEC and GPS-STEC. The parameters of the perturbation surface are optimized such that the resultant $3 \mathrm{D}$ electron density distribution is in compliance with the STEC estimates obtained from a GPS satellitereceiver network in the region. Also, instead of bounding the perturbation surfaces, perturbed critical frequency and maximum ionization height surfaces are bounded using a mathematical function that models the physical behavior of ionosphere for a midlatitude region. In order to decrease the computational cost, precomputed electron density matrices are used for IRI-PLAS-STEC calculation. The perturbation surface parameters are optimized using Particle Swarm Optimization (PSO). The reduced number of optimization parameters due to the planar perturbation surfaces, using precomputed electron density voxel values for model based STEC calculation and the use of bounding functions for modeling the physical limits of ionosphere parameters not only provide faster convergence and reduces the computational complexity but also increases the reliability and accuracy of the reconstruction. The comparisons with ionosonde electron density distributions over Turkey and Central Europe indicate that IONOLAB-CIT is a reliable tool in reconstruction of electron density both for disturbed and nondisturbed days. 


\section{RAY TRACING IN IONOSPHERE: IONOLAB-RAY}

IONOLAB-RAY is an user-friendly radio propagation software for tracing of propagation of electromagnetic waves through ionosphere which is based on ray tracing methods that are operated in a spherical voxel structure between $100 \mathrm{~km}$ to $20.000 \mathrm{~km}$ [18], [19]. The anisotropic, inhomogeneous and time varying ionosphere is simulated using the IRI-Plas as the background model. Refractive index of each voxel is calculated by Appleton-Hartree formula, which includes electron cyclotron frequency, collision frequency and anisotropicity due to geomagnetic field. IONOLAB-RAY allows the automatic input of GPS/GIM-TEC, and thus, update of the ionosphere to the current geomagnetic conditions. This capability has important impact in characterization of ionosphere during geomagnetic storm conditions. IONOLAB-RAY can be used to simulate typical $\mathrm{HF}$ and satellite communication scenarios. One important capability of IONOLAB-RAY is the computation of group delay through ordinary and extraordinary paths of low and high rays. The Faraday Rotation can also be computed at the possible receiver locations. IONOLAB-RAY provides a reliable and accurate electromagnetic wave simulation environment for any ionospheric condition and any ray path over the earth [18], [19].

\section{CONCLUSION}

This paper briefly reviews the online SW services of IONOLAB group provided at www.ionolab.org for robust, reliable and accurate estimate of GPS-TEC as IONOLABTEC; online computation of ionospheric climatic model, IRIPlas; 2-D ionospheric maps of GIM-TEC, foF2, hmF2 and Windex as IRI-PLAS-MAP; and STEC computation using IRIPlas as IRI-PLAS-STEC.

IONOLAB group is also active in development of 2-D and 3-D imaging tools for ionospheric electron density reconstruction and TEC mapping as IONOLAB-CIT and IONOLAB-MAP, and ray tracing in time varying, inhomogeneous and anisotropic ionosphere as IONOLABRAY. All of the above mentioned algorithms are developed using the research grants by TUBITAK and they can be used by researchers world-wide for scientific and academic purposes.

\section{Acknowledgment}

The IONEX files that include GIM-TEC, Satellite DCB and ephemeris data that are used in computation of IONOLAB products are obtained from IGS Iono Working Group Data Analysis Center of Jet Propulsion Laboratory at ftp://cddis.gsfc.nasa.gov/pub/gps/products/ionex/.

\section{References}

[1] T.L. Gulyaeva, F. Arikan, I. Stanislawska, and L.V. Poustovalova, "Global distribution of zones of enhanced risk for the ionospheric weather," Journal of Geography, Environment and Earth Science
International, vol. 4, no 1, pp. 1-13, 2016 doi:10.9734/JGEESI/ 2016/20488.

[2] F. Arikan, U. Sezen, O. Arikan, O. Ugurlu, H. Nayir, "Space weather activities of IONOLAB group: IONOLAB-TEC," In EGU General Assembly Conference Abstracts, vol 11, p. 5188, April 2009.

[3] B. Aktug, O. Lenk, M. Kurt, E. Parmaksiz, S. Ozdemir, F. Arikan, U. Sezen, C. Toker, O. Arikan, "Space weather activities of IONOLAB group using TNPGN GPS network," In IEEE 5th International Conference on Recent Advances in Space Technologies (RAST), pp. 604-607, June 2011.

[4] F. Arikan, C. B. Erol, O. Arikan, "Regularized estimation of vertical total electron content from Global Positioning System data," Journal of Geophysical Research: Space Physics, vol. 108(A12), December 2003.

[5] H. Nayir, F. Arikan, O. Arikan, C. B. Erol, "Total electron content estimation with Reg-Est," Journal of Geophysical Research: Space Physics, vol. 112(A11), November 2007.

[6] U. Sezen, F. Arikan, O. Arikan, O. Ugurlu, A. Sadeghimorad, "Online, automatic, near-real time estimation of GPS-TEC: IONOLAB-TEC," Space Weather, vol. 11, no. 5, pp. 297-305, May 2013. doi:10.1002/swe.20054.

[7] F. Arikan, H. Nayir, U. Sezen, O. Arikan, "Estimation of single station interfrequency receiver bias using GPS-TEC," Radio Science, vol. 43 no 4, August 2008.

[8] D. Bilitza, L. A. McKinnell, B. Reinisch, T. Fuller-Rowell, "The International Reference Ionosphere (IRI) today and in the future," $\mathrm{J}$. Geodesy, vol. 85, no. 12, pp. 909-920, Nov. 2011. doi: 10.1007/s00190010-0427-x.

[9] T. L. Gulyaeva, D. Bilitza, "Towards ISO Standard Earth Ionosphere and Plasmasphere Model, In: New Developments in the Standard Model," edited by R.J. Larsen, pp. 1-39, NOVA, Hauppauge, New York.

[10] T. L. Gulyaeva, F. Arikan, I. Stanislawska, "Inter-hemispheric imaging of the ionosphere with the upgraded IRI-Plas model during the space weather storms," Earth Planets Space, vol. 63, no. 8, pp. 929-939, 2011. doi: 10.5047/eps.2011.04.007.

[11] F. Arikan, U. Sezen, T. L. Gulyaeva, O. Cilibas, "Online, automatic, ionospheric maps: IRI-PLAS-MAP," Advances in Space Research, vol. 55, no. 8, pp. 2106-13, April 2015. doi:10.1016/j.asr.2014.10.016.

[12] H. Tuna, O. Arikan, F. Arikan, T. L. Gulyaeva, U. Sezen, "Online user-friendly slant total electron content computation from IRI-Plas: IRI-Plas-STEC," Space Weather, vol. 12, no. 1, pp. 64-75, January 2014.

[13] F. Arikan, S. Shukurov, H. Tuna, O. Arikan, T. L. Gulyaeva, "Performance of GPS slant total electron content and IRI-Plas-STEC for days with ionospheric disturbance," Geodesy and Geodynamics, online March 2016. doi:10.1016/j.geog.2015.12.009.

[14] M. N. Deviren, F. Arikan, O. Arikan, "Automatic regional mapping of total electron content using a GPS sensor network and isotropic universal Kriging," In IEEE 16th International Conference on Information Fusion (FUSION 2013), pp. 1664-1669, July 2013.

[15] I. Sayin, F. Arikan, O. Arikan, "Regional TEC mapping with Random Field Priors and Kriging," Radio Science, vol. 43, no.5, October 2008. doi:10.1029/2007RS003786.

[16] O. Erturk, O. Arikan, F. Arikan, "Tomographic reconstruction of the ionospheric electron density as a function of space and time," Advances in Space Research, vol. 43, no. 11, pp. 1702-1710, June 2009.

[17] H. Tuna, O. Arikan, F. Arikan, "Regional model-based computerized ionospheric tomography using GPS measurements: IONOLAB-CIT," Radio Science. vol. 50, no. 10, pp. 1062-75, October 2015.

[18] E. Erdem, F. Arikan, M. N. Deviren, I. Cor, "A model based ray tracing algorithm for anisotropic and inhomogeneous ionosphere with GIMTEC assimilation," In IEEE 7th International Conference on Recent Advances in Space Technologies (RAST), pp. 477-481, June 2015.

[19] E. Erdem, F. Arikan, "IONOLAB-RAY: A wave propagation algorithm for anisotropic and inhomogeneous ionosphere," under review in Turkish Journal Of Electrical Engineering \& Computer Sciences, ELK1602-119, March 2016. 